Results Tregs from SLE patients showed a significantly reduced number,elevated apoptosis rates and impared suppressive capacity compared with NCs. The increased Tregs apoptosis was negatively correlated with the total number of Tregs and positively correlated with disease activities.Microarray profiles of Tregs from SLE subjects reveal a cellular response that could make the cells sensitive to apoptosis,partially due to the stress responses,DNA-damaging and cytokine stimulation.

Conclusions This global picture of pathway-specific expression signatures is a step further dissecting Treg cells defects in the pathogenesis of SLE, and may shed light on the newly therapeutic strategies towards the aberrant Tregs apoptosis and reconstruction of SLE immune homeostasis.

\section{THE PATHOGENIC RELEVANCE OF T FOLLICULAR HELPER CELLS-PLASMABLASTS AXIS IN PATIENTS WITH SYSTEMIC LUPUS ERYTHEMATOSUS}

S Nakayamada*, S Kubo, M Yoshikawa, Y Miyazaki, K Sakata, K Nakano, S Iwata, I Miyagawa, K Saito, Y Tanaka. University of Occupational and Environmental Health, First Department of Internal Medicine, Kitakyushu, Japan

\subsection{6/lupus-2017-000215.52}

Background and aims The aim of this study was to assess the peripheral immune cell phenotypes in a correlation with clinical findings in patients with systemic lupus erythematosus (SLE).

Methods Peripheral blood mononuclear cells were obtained from 143 SLE patients and 26 healthy donors (HD). Circulating $\mathrm{B}, \mathrm{T}$ and dendritic cells were defined based on flow cytometric analysis for human immune system termed "the Human Immunology Project" proposed by the National Institutes of Health (NIH) and the Federation of Clinical Immunology Societies (FOCIS).

Results The proportions of $\mathrm{CD}^{+}{ }^{+} \mathrm{CD} 4^{+} \mathrm{CXCR}^{+} \mathrm{ICOS}^{+} \mathrm{T}$ follicular helper (Tfh) cells, but not $\mathrm{CD}^{+} \mathrm{CD} 4^{+} \mathrm{CXCR} 3^{+} \mathrm{CCR} 6$ Th1 and $\mathrm{CD}^{+}{ }^{+} \mathrm{CD} 4^{+} \mathrm{CXCR} 3{ }^{-} \mathrm{CCR} 6{ }^{+}$Th17 cells, were higher in SLE than the HD. The proportions of $\mathrm{CD}_{1}{ }^{+}$ $\mathrm{CD}_{20}{ }^{+} \mathrm{IgD}^{+} \mathrm{CD} 27^{+}$central memory $\mathrm{B}$ cells and $\mathrm{CD} 19^{+} \mathrm{CD} 20^{+} \mathrm{IgD}^{-} \mathrm{CD} 27^{-}$effector $\mathrm{B}$ cells were higher in SLE. The largest difference relative to the $\mathrm{HD}$ was observed in the proportion of $\mathrm{CD} 19^{+} \mathrm{CD} 20^{-} \mathrm{CD} 27^{+} \mathrm{CD} 38^{+}$plasmablasts, which was higher in SLE and correlated with BILAG index. The proportion of Tfh cells correlated with serum IgG level, and the proportion of activated Tfh cells correlated with serum anti-Sm antibody level. Among helper T cell subsets (Th1, Th17, Treg and Tfh), Tfh cells only showed positive correlation with the proportion of plasmablast $(\mathrm{r}=0.24, \mathrm{p}=0.02)$.

Conclusions Peripheral immuno-phenotyping confirmed the importance of Tfh-plasmablasts axis in patients with SLE, i.e. activation of Tfh cells correlated with autoantibody production while plasmablast did with disease activity of SLE. Our findings supported the relevance of Tfh-plasmablasts axis as a potential therapeutic target for SLE.

\section{REPOSITORY CORTICOTROPIN INJECTION EXERTS DIRECT ACUTE EFFECTS ON HUMAN B CELL GENE EXPRESSION DISTINCT FROM THE ACTIONS OF GLUCOCORTICOIDS}

${ }^{1} \mathrm{~N}$ Olsen*, ${ }^{1} \mathrm{~A}$ Benko, ${ }^{1} \mathrm{C}$ McAloose, ${ }^{2} \mathrm{~T}$ Sunyer, ${ }^{2} \mathrm{P}$ Becker, ${ }^{1} \mathrm{~W}$ Kovacs. ${ }^{1}$ Penn State Hershey, Medicine, Hershey, USA; ${ }^{2}$ Mallinckrodt Pharmaceuticals, Clinical Translational Research, Hazelwood Missouri, USA

\subsection{6/lupus-2017-000215.53}

Background and aims Repository corticotropin injection (RCI; H.P. Acthar Gel) is a porcine pituitary-derived ACTH preparation approved by the FDA for therapy in selected cases of SLE. Previous studies have shown that RCI directly inhibits human B cell function in vitro.

Methods We used RNA-Seq to identify elements of the transcriptome that are acutely modulated by RCI in human B cells activated in vitro by IL4 and CD40 ligand. We compared RCI effects to those of a synthetic glucocorticoid (dexamethasone; Dex) under the same conditions.

Results 115 unique gene transcripts were significantly and reproducibly upregulated by RCI after 24 hours in culture. Pathways analysis revealed that upregulated genes were overrepresented in "immune system response" $(2.8$-fold; $\mathrm{p}=0.026)$ and "response to stress" $(4.16$-fold; $p=0.0069) .74$ gene transcripts were down-regulated by RCI, and these were over-represented in two pathways: "immune system response" (2.91fold; $p=0.035)$ and "cellular process" (1.73-fold; $p=0.0036)$. In Dex-treated cells, 65 gene transcripts were upregulated and 23 gene transcripts were down-regulated. There was no overlap between the set of genes upregulated by RCI and Dex. Two genes (PARM1 and RANKL) were downregulated by both RCI and Dex. Pathways analysis of Dex- treated samples did not reveal significant overrepresentation of regulated genes in any specific ontologic pathway.

Conclusions These data suggest that RCI exerts direct effects on human B cells to acutely modulate gene expression. These effects are distinct from those of glucocorticoids, supporting potential differences in mechanism of action of these two agents for treatment of autoimmune diseases.

\section{$54 \quad$ R052 AUTOANTIBODIES ARISE FROM SELF-REACTIVE PROGENITORS IN A MOTHER OF A CHILD WITH NEONATAL LUPUS}

1J Reed*, ${ }^{2} \mathrm{M}$ Gorny, ${ }^{2} \mathrm{~L} \mathrm{Li},{ }^{3} \mathrm{~T}$ Cardozo, ${ }^{4} \mathrm{~J}$ Buyon, ${ }^{4} \mathrm{R}$ Clancy. ${ }^{1}$ Garvan Institute of Medical Research, Immunology, Darlinghurst, Australia; ${ }^{2}$ New York University School of Medicine, Pathology, New York, USA; ${ }^{3}$ New York University School of Medicine, Pharmacology, New York, USA; ${ }^{4}$ New York University School of Medicine, Medicine, New York, USA

\subsection{6/lupus-2017-000215.54}

Background and aims Autoantibodies targeting Ro52 occur in systemic lupus erythematosus, Sjogren's syndrome and idiopathic inflammatory myopathies. Yet the most compelling evidence for their pathogenesis is the development of cardiac conduction abnormalities, a manifestation of neonatal lupus, in 
foetuses exposed to maternal anti-Ro52 autoantibodies. Recent studies investigating other pathogenic autoantibodies (antiinterferon, anti-desmoglein) report that they arise as a result of somatic mutation. The aim of this study was to determine how anti-Ro52 autoantibodies originate.

Methods We traced the evolution of two anti-Ro52 autoantibodies isolated from circulating IgG-switched memory B-cells from a mother of two children with cardiac neonatal lupus. Each antibody was expressed as its immune form or preimmune ancestor by reverting somatic mutations to germline sequence. Antibody reactivity against autoantigens Ro52, Ro60, La and dsDNA were tested by ELISA.

Results Both anti-Ro52 autoantibodies utilised the same heavy and light chain genes (IGHV3-23 and IGLV1-44) but represented distinct clones based on differing complementarity determining region sequences. Anti-Ro52 autoantibodies exhibited a low frequency $(3 \%-4 \%)$ of somatic mutations compared to the average rate of $8 \%$ in healthy switched memory B-cells. In contrast to other pathogenic autoantibodies, the preimmune (germlined) anti-Ro52 autoantibodies showed specific binding to Ro52. However, Ro52 reactivity was higher for the mutated post-immune antibodies compared to their preimmune counterparts demonstrating that autoreactivity was enhanced by affinity maturation.

Conclusions These data demonstrate that Ro52 reactivity is an intrinsic property of the germline antibody repertoire in a mother of children affected by neonatal lupus and indicate defects in central and peripheral tolerance pathways allow propagation of pathogenic autoantibodies.

\section{RESPONSE GENE TO COMPLEMENT-32 PROMOTES PLASMA CELL DIFFERENTIATION AND ENHANCES LUPUS-LIKE CHRONIC GRAFT VERSUS HOST DISEASE}

${ }^{1} \mathrm{H}$ Rus*, ${ }^{1} \mathrm{~A}$ Tatomir, ${ }^{2} \mathrm{~V}$ Nguyen, ${ }^{3} \mathrm{C}$ Cudrici, ${ }^{4} \mathrm{~T}$ Badea, ${ }^{2} \mathrm{~V}$ Rus. ${ }^{1}$ University of Maryland School of Medicine, Neurology, Baltimore, USA; ${ }^{2}$ University of Maryland School of Medicine, Medicine, Baltimore, USA; ${ }^{3}$ National Institute of Health, NIAMS, Bethesda, USA; ${ }^{4}$ National Institute of Health, NEI, Bethesda, USA

\subsection{6/lupus-2017-000215.55}

Background and aims Response Gene to Complement (RGC) -32 plays an important role in cell cycle activation. Our prior studies showed that RGC-32 promotes Th17 differentiation of CD4 T cells. We used wild-type (WT) and RGC-32 knockout (KO) mice to determine whether lack of RGC-32 impairs B cell differentiation and activation and alters autoimmune parameters in the chronic graft versus host disease (cGVHD) model of lupus.

Methods TLR-dependent and $\mathrm{T}$ dependent $\mathrm{B}$ cell differentiation to plasma cells (PC) was induced with LPS and with $\mathrm{CD} 40 \mathrm{mAb}$ plus IL-4. cGVHD was induced with $100 \times 10^{6} \mathrm{Bm} 12$ splenocytes injected into WT or RGC-32 KO recipients.

Results RGC-32 KO B cells failed to differentiate normally to PC as demonstrated by a 2 -fold reduction in PC numbers generated after stimulation and impaired upregulation of $\operatorname{Prdm} 1$ and IRF4 mRNA. RGC-32 transcripts were upregulated in spleen cells from cGVHD mice and protein expression was detected in B cells and germinal centre (GC) cells. RGC-32 $\mathrm{KO}$ hosts displayed an attenuated autoimmune phenotype as demonstrated by decreased production of anti-dsDNA autoantibodies and proliferation of germinal centre $\mathrm{B}$ cells. In addition a decreased number of IgG anti-dsDNA secreting PC and IRF4 and Prdm1 mRNA expression were found

Conclusions These results suggest that expression of RGC-32 in B cells is critical for optimal GC proliferation, PC differentiation and autoantibody production in a murine model of lupus. These data support the idea that RGC-32 blockade has the potential to attenuate autoimmune parameters of cGVHD and possibly reverse abnormalities in the $\mathrm{T}$ and $\mathrm{B}$ cell that contribute to lupus pathogenesis.

\section{MICROMANAGING LUPUS NEPHRITIS: MIR17-92 MODULATES REGULATORY T CELL ACTIVITY BY TARGETING FOXP3 CO-REGULATORS}

${ }^{1} \mathrm{HY}$ Yang, ${ }^{2} \mathrm{CY}$ Wu, ${ }^{2} \mathrm{~L}$ Huang. ${ }^{1}$ Chang-Gung Memorial Hospital- Linko Branch, Nephrology, Taoyuan, Taiwan R.O.C; ${ }^{2}$ Chang-Gung Memorial Hospital- Linko Branch, Paediatrics, Taoyuan, Taiwan R.O.C

\subsection{6/lupus-2017-000215.56}

Background and aims Regulatory $\mathrm{T}$ (Treg) cells play a critical role in maintaining self-tolerance and controlling the magnitude of physiologic immune response. The Treg transcription factor forkhead box P3 (Foxp3) works in concert with other co-regulator molecules including Eos to determine suppressive phenotype of Treg. We identified miR17-92 cluster targeting Eos through bioinformatics approaches.

Methods We generated T-cell-specific miR-17-92 null (mir1792 -/-) mice by mating mir17-92flox/flox mutants to CD4-Cre + transgenic mice. Treg from mir17-92 -/- mice will be isolated, followed by suppression assay to evaluate the role of the miR-17-92 cluster in Treg function. We applied pristane to induce lupus nephropathy in wild type and mir17-92 -/mice. We examined the up-stream promoter region of miR17-92 for binding sites of down-stream mediators of IL-6 signalling, verified by chromatin immunoprecipitation assay.

Results The inflammatory cytokine IL-6 unregulated miR17-92 through HIF-1. MiR17-92 cluster.actively suppressed Eos expression. Knockdown of miR17-92 in Treg enhanced their suppressive activity. Mir17-92 T cell specific deficiency mitigated pristane induced-lupus nephropathy associated with diminished Th17 cells and autoantibody. Moreover, histological analysis revealed a lower mean renal histopathology score and less compliment deposition. Ectopic expression of miR-17 downmodulated the suppression functions of Tregs and provided Treg with partial effector activity via the derepression of cytokine genes.

Conclusions Our studies suggest that miR17-92 modulates Treg cell function by targeting Eos and potentially additional Foxp3 co-regulators, unveiling the future therapeutic potential of microRNA manipulation in lupus nephritis.

\section{A HIGHER FREQUENCIES OF T HELPER 22 CELLS IN PATIENTS WITH NEW ONSET ACTIVE SYSTEMIC LUPUS ERYTHEMATOSUS}

'W Zhong*, 'L Zhao, ' 'Z Jiang, ${ }^{2} Y$ Jiang. ${ }^{1}$ First Hospital of Jilin University, Rheumatology, Changchun, China; ${ }^{2}$ First Hospital of Jilin University, Central Laboratory, Changchun, China

\subsection{6/lupus-2017-000215.57}

Background and aims This study is aimed at elucidating the potential role of Th22 cells in patients with SLE. 